\title{
Concurrent chemoradiotherapy for organ function preservation in advanced patients with hypopharyngeal and laryngeal cancer
}

\author{
YASUNAO KOGASHIWA, KOHICHI YAMAUCHI, HIROSHI NAGAFUJI, TAKEHIRO MATSUDA, \\ TOSHIHITO TSUBOSAKA, TOMOKO TSUTSUMI, TAKEHIRO KARAHO and NAOYUKI KOHNO \\ Department of Otolaryngology, Head and Neck Surgery, Kyorin University \\ School of Medicine, 6-20-2 Shinkawa, Mitaka, Tokyo 181-8611, Japan
}

Received March 4, 2009; Accepted June 1, 2009

DOI: $10.3892 /$ or_00000550

\begin{abstract}
Preservation of the larynx is the most critical factor influencing quality of life in the treatment of head and neck cancer. This clinical study focuses on laryngeal functionpreserving chemoradiotherapy for locally advanced hypopharyngeal and laryngeal cancer. Thirty-two resectable cases with histologically proven squamous cell carcinoma undergoing function-preserving therapy were examined. Induction chemotherapy comprised cisplatin and 5-fluorouracil, and another cycle of chemotherapy was performed for responders. Chemoradiotherapy comprised conventional irradiation and weekly chemotherapy (nedaplatin plus docetaxel). Nonresponder patients were excluded from further chemotherapy and were changed to other surgical treatment. Three patients were non-responders for induction chemotherapy, and 29 patients were treated with chemoradiotherapy. Thus, 21 out of 29 patients obtained preserved laryngeal function. Initial larynx preservation rate with these treatment strategies was $93.8 \%$. This study provides a new concept for laryngeal function-preserving treatment that should be considered for locally advanced laryngeal and hypopharyngeal cancer.
\end{abstract}

\section{Introduction}

In the treatment of head and neck cancer, particularly for hypopharyngeal and laryngeal cancer, preservation of the larynx is the most critical factor influencing quality of life (QOL). In cases of early laryngeal cancer, this aim is relatively easy to achieve by radiation and laser treatment $(1,2)$. In contrast, treatment for locally advanced cases requires some modification. Recently, results of comparative studies on combined chemotherapy and radiotherapy have reported significantly improved laryngeal preservation rates and local

Correspondence to: Dr Naoyuki Kohno, Department of Otolaryngology, Head and neck Surgery, Kyorin University School of medicine, 6-20-2 Shinkawa, Mitaka, Tokyo 181-8611, Japan E-mail: naoyukikohno@yahoo.co.jp

Key words: nedaplatin, docetaxel, induction chemotherapy, concurrent chemoradiotherapy, phase I study control rates compared with conventional radiotherapy alone. Progress in combination therapy for advanced laryngeal cancer is required to reduce the need for surgical therapy (3).

Loss of the larynx is sometimes unavoidable in surgery. However, preservation of laryngeal function has been made increasingly possible by surgical treatment, and indications for such procedures have been expanded even to the point of salvage surgery for recurrent cases (4-6). Herein, we report on a clinical study which focused on laryngeal functionpreserving chemoradiotherapy for locally advanced hypopharyngeal and laryngeal cancer.

\section{Materials and methods}

Subjects comprised 134 patients with hypopharyngeal or laryngeal cancer who received the first treatment in our department between January 2003 and December 2008. Of these, 47 patients were treated according to the protocol shown in Table I. Tumour was classified as unresectable in 15 patients. Criteria for unresectability included: $>180^{\circ} \mathrm{C}$ encasement of the carotid artery; fixation to prevertebral fascia; massive $(>8 \mathrm{~cm}$ ) fixed soft-tissue disease; and gross extension along the pharyngeal wall. Thus, in this study, 32 resectable cases with histologically proven squamous cell carcinoma of the hypopharynx and larynx undergoing function-preserving therapy were examined. All patients were provided written informed consent before enrolment in the study. All patients were required to have an Eastern Cooperative Oncology Group (ECOG) criteria performance status of less than I and bidimensionally measurable disease.

Initial evaluation included complete physical examination, blood cell count, liver biochemistry, chest radiography, and oropharyngolaryngeal fiberoptic examination. Patients were required to display normal renal function (blood urea nitrogen (BUN) $<20 \mathrm{mg} / \mathrm{dl}$; serum creatinine $<1.5 \mathrm{mg} / \mathrm{dl}$ ) and hepatic function (serum glutamic oxaloacetic transaminase (SGOT) $<40 \mathrm{U} / \mathrm{dl}$; serum glutamic pyruvate transaminase (SGPT) $<40 \mathrm{U} / \mathrm{dl}$; bilirubin $<1.5 \mathrm{mg} / \mathrm{dl}$ ). Patients $>80$-yearsold were excluded. Patient characteristics are shown in Table II. Staging classification was performed according to the International Union Against Cancer staging system.

Induction chemotherapy comprised cisplatin (CDDP) $\left(80 \mathrm{mg} / \mathrm{m}^{2} / 2-\mathrm{h}\right.$ infusion on day 1$)$ and 5 -fluorouracil (5FU) $\left(800 \mathrm{mg} / \mathrm{m}^{2} / 24-\mathrm{h}\right.$ infusion on days $\left.1-5\right)$. Another cycle of chemotherapy was performed for responders ( $>80 \%$ response) 
Table I. The treatment program of hypopharyngeal and laryngeal cancer.

\begin{tabular}{|c|c|c|}
\hline Resectable (32) $)^{\mathrm{a}}$ & & \\
\hline Supraglottic & & \\
\hline$<\mathrm{T} 2: \mathrm{IC}(11)^{\mathrm{a}}$ & Responder (11) ${ }^{\mathrm{a}}$ & CRT \\
\hline & Non-responder $(0)^{\mathrm{a}}$ & Surgery \\
\hline Glottic & & \\
\hline $\mathrm{T} 3,4: \mathrm{CRT}(5)^{\mathrm{a}}$ & & \\
\hline$<\mathrm{N} 2: \mathrm{IC}(1)^{\mathrm{a}}$ & Responder (0) & CRT \\
\hline & Non-responder (1) ${ }^{\mathrm{a}}$ & Surgery \\
\hline Subglottic & & \\
\hline$<\mathrm{T} 2: \mathrm{IC}(0)^{\mathrm{a}}$ & Responder $(0)^{\mathrm{a}}$ & CRT \\
\hline & Non-responder $(0)^{\mathrm{a}}$ & Surgery \\
\hline Hypopharyngeal & & \\
\hline$<\mathrm{T} 2: \mathrm{IC}(15)^{\mathrm{a}}$ & Responder (13) ${ }^{\mathrm{a}}$ & CRT \\
\hline & Non-responder $(2)^{\mathrm{a}}$ & Surgery \\
\hline Unresectable & & \\
\hline $\operatorname{CRT}(15)^{\mathrm{a}}$ & & \\
\hline
\end{tabular}

${ }^{a}$ Number of patients. CRT, concurrent chemoradiotherapy.

Table II. Patient characteristics.

\begin{tabular}{lc}
\hline Evaluable patients & 32 \\
Male & 30 \\
Female & 2 \\
Mean age (range) & $65.5(48-80)$ \\
PS 0/1 & $25 / 7$ \\
Site of primary & \\
$\quad$ Larynx & 17 \\
Hypopharynx & 15 \\
\hline
\end{tabular}

at 3-week intervals. Chemoradiotherapy comprised conventional irradiation with $2.0 \mathrm{~Gy} /$ day for 5 days/week up to a total dose between 60 and $68 \mathrm{~Gy}$ and nedaplatin $15 \mathrm{mg} / \mathrm{m}^{2} /$ 1-h infusion on days $1,8,15,22,29$ and $36\left(3 \mathrm{mg} / \mathrm{m}^{2} 3\right.$ times/ week up to 6 weeks for the initial 4 patients) plus docetaxel $10 \mathrm{mg} / \mathrm{m}^{2} / 2$-h infusion on days $1,8,15,22,29$ and 36 . Patients with non-responsive tumour and defined progressive disease or with cumulative toxicity exceeding the benefits of further drug administration were excluded from further chemotherapy and were changed to other surgical treatment.

Response to the treatment was assessed as the decrease in total tumour area measured directly with callipers or through radiological features. Complete response (CR) was defined as disappearance of all measurable and assessable disease for a minimum duration of 4 weeks. Partial response (PR) was defined as a $\geq 50 \%$ reduction in sum of the products of perpendicular diameters of all measured lesions without an
Table III. Results of the chemoradiotherapy.

\begin{tabular}{|c|c|c|c|c|}
\hline \multicolumn{5}{|l|}{ CR: $23 / 29$} \\
\hline AOD & & & & 15 \\
\hline AWD & & & & 1 \\
\hline Relapse & SCL-CHEP & Total & DWD & 1 \\
\hline Relapse & Total & AWD & & 1 \\
\hline Relapse & $\begin{array}{l}\text { Endolaryngeal } \\
\text { laser surgery }\end{array}$ & Total & AOD & 1 \\
\hline Relaplse & DWD & & & 2 \\
\hline DOD & & & & 2 \\
\hline \multicolumn{5}{|l|}{ PR: 4/29 } \\
\hline SCL-CHEP & DWD & & & 1 \\
\hline $\begin{array}{l}\text { Rejected case of } \\
\text { salvage surgery }\end{array}$ & DWD & & & 1 \\
\hline $\begin{array}{l}\text { Rejected cases of } \\
\text { salvage surgery }\end{array}$ & AWD & & & 2 \\
\hline \multicolumn{5}{|l|}{$\mathrm{NC}: 1 / 29$} \\
\hline $\begin{array}{l}\text { Rejected case of } \\
\text { salvage surgery }\end{array}$ & DWD & & & 1 \\
\hline \multicolumn{5}{|l|}{ PD: $1 / 29$} \\
\hline SCL-CHEP & AOD & & & 1 \\
\hline
\end{tabular}

AOD, alive without disease; AWD, alive with disease; DWD, died with disease; DOD, died by other disease; Total, total laryngectomy.

increase in size of any lesion or appearance of new lesions for $\geq 4$ weeks. Minimal response (MR) was defined as a decrease of $25-50 \%$. No change (NC) was defined as a $<25 \%$ change in sum of the products of the perpendicular diameter of all measured lesions. Progressive disease (PD) was defined as an increase of $\geq 25 \%$ in the sum of products of perpendicular diameters of all measured lesions from the point of maximal response or appearance of new lesions. Patients who did not meet the criteria for PR or CR (i.e., displaying NC, MR or $\mathrm{PD})$ were classified as non-responders.

\section{Results}

Table I shows the number of patients of this study. For induction chemotherapy, CR and PR were found in 8 and 19 cases, respectively. Among 3 non-responders, partial laryngectomy was indicated in 1 patient and total laryngectomy was indicated in 2 patients. The overall survival rate of evaluable 32 patients is shown in Fig. 1. The 36 month survival rate of laryngeal cancer and hypopharyngeal cancer, and the total was $82.9,51.6$ and $67.1 \%$ respectively.

The 24 responders with induction chemotherapy and 5 glottic T3 and T4 cancer patients were treated with chemoradiotherapy. Twenty patients $(69.0 \%)$ received the full treatment as planned, 29 (100\%) received full-dose radiotherapy and $20(69.0 \%)$ received full-dose chemotherapy. On the other hand, 8 patients were given $\geq 80 \%$ of chemotherapy doses and 1 patient received $60 \%$. The 29 patients were 
overall survival of 32 evaluable patients
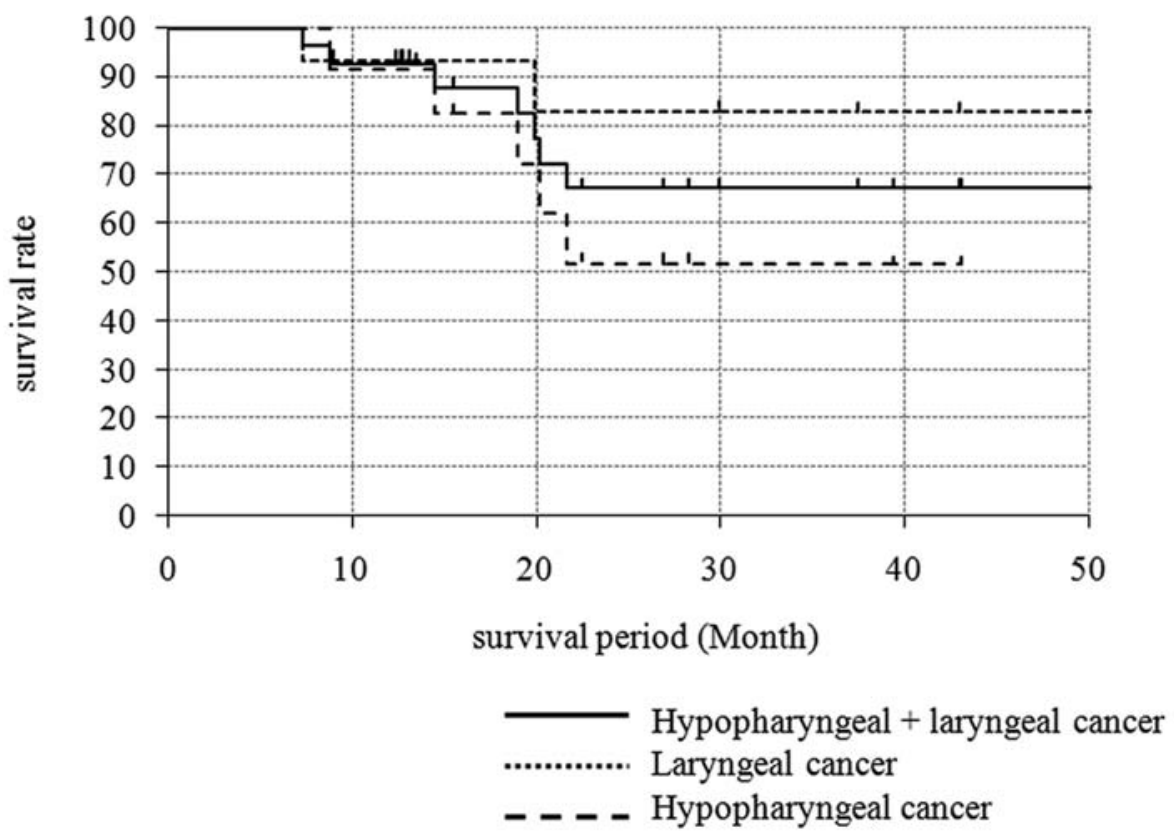

Figure 1. The overall survival rate of evaluable 32 patients.

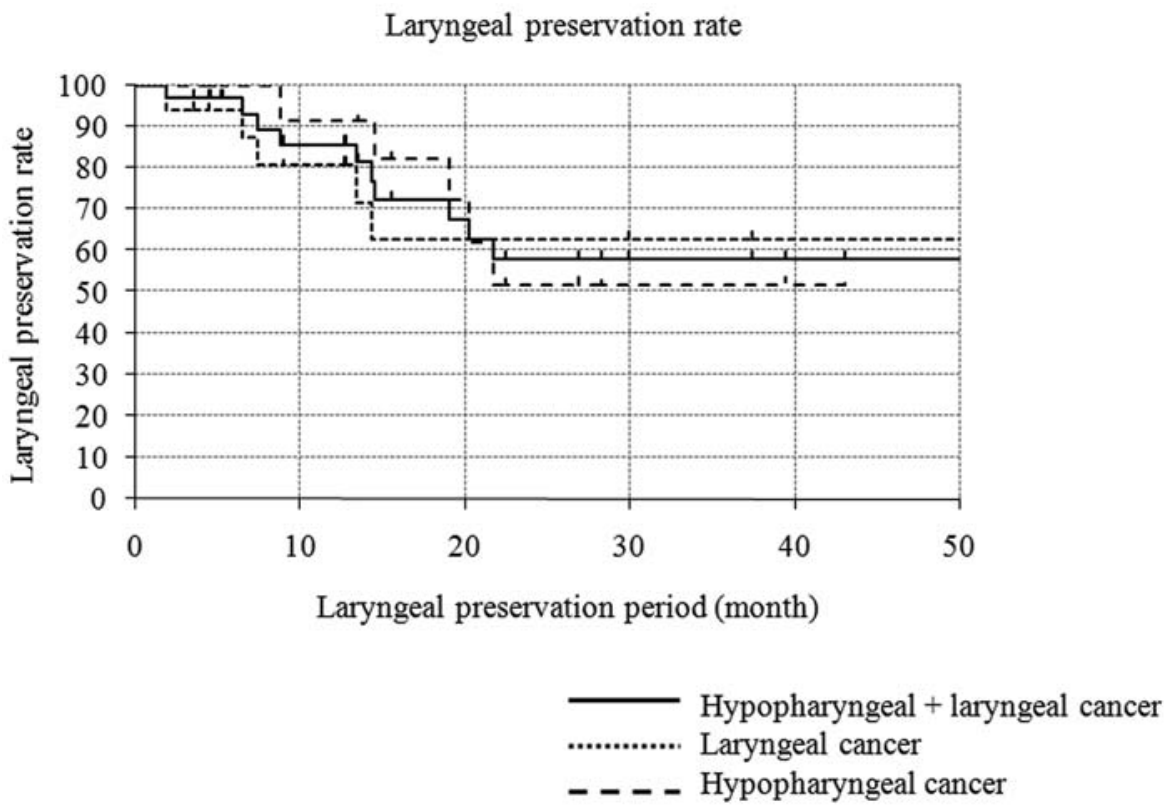

Figure 2. Laryngeal preservation rate of chermoradiotherapy.

evaluable for response achieved 23 CR (79.3\%), 4 PR (13.8\%), $1 \mathrm{NC}(3.4 \%)$, and $1 \mathrm{PD}(3.4 \%)$, with an overall response rate of $93.1 \%$. For $1 \mathrm{CR}, 1 \mathrm{PR}$ and $1 \mathrm{PD}$ patient, supracricoid laryngectomy with cricohyoidoepiglottopexy (SCL-CHEP) was performed but 2 out of 3 relapsed. One CR and 2 relapsed patients underwent total laryngectomy. Four patients rejected salvage surgery. Two have died and 2 are alive with disease. Initial larynx preservation rate with these treatment strategies was thus $93.8 \%$ (30/32). For chemo- radiotherapy, 19 of 29 patients $(65.5 \%)$ obtained preserved laryngeal function (Table III). The laryngeal preservation rate is shown in Fig. 2. In laryngeal cancer, the 48 month laryngeal preservation rate was $67.2 \%$. On the other hand, in hypopharyngeal cancer the 36 month laryngeal preservation rate was $51.5 \%$. In total the 36 month laryngeal preservation rate was $57.8 \%$.

Adverse events are shown in Table IV. Critical adverse events at grade III/IV included 8 cases of neutropenia and 2 
Table IV. Toxicities with chemoradiotherapy (29 evaluable patients).

\begin{tabular}{lcc}
\hline & Grade I/II & Grade III/ IV \\
\hline Nausea or vomiting & 8 & \\
Anemia & 4 & \\
Leukopenia & 6 & 6 \\
Neutropenia & 5 & 8 \\
Mucositis & 20 & 2 \\
Diarrhea & 6 & \\
Skin & 12 & 2 \\
Allergic reaction & 6 & \\
\hline
\end{tabular}

cases of stomatitis. Anaphylactic symptoms appeared $30 \mathrm{~min}$ after nedaplatin administration in 3 cases (rash and mild seizure in 2 cases each).

\section{Discussion}

In addition to improved survival rates, preservation of the larynx is an important issue in the treatment of laryngeal and hypopharyngeal cancer. In resectable cases, options include surgery, radiotherapy, and concurrent chemoradiotherapy. Forastiere et al performed a comparative trial for advanced laryngeal cancer and reported excellent results in which chemoradiotherapy yielded a 2-year laryngeal preservation rate of $88 \%$ and a local control rate of $78 \%$. Moreover, additional chemotherapy reduced distant metastases (2-year distant metastasis rates: induction chemotherapy, $9 \%$; concurrent chemoradiotherapy, $8 \%$; and radiotherapy alone, $16 \%)$. Efficacy of concurrent chemotherapy was described in terms of laryngeal preservation and survival periods (3). On the other hand, laser treatment was also suggested for partial and subtotal laryngectomy to preserve larynx, as well as hyperfraction radiotherapy which might be effective. Salvage total laryngectomy after irradiation or partial resection was performed in 151 of 734 cases of laryngeal and hypopharyngeal cancer, and 5- and 10-year survival rates were 55 and $39 \%$, respectively (7). Fowler et al reported that salvage surgery resulted in 65.2 and $37.7 \%$ for 5 - and 10 -year survival rates, respectively, including 53 cases of radiation failure, 6 cases of postoperative recurrence, and 5 cases of recurrence after surgery and irradiation (8).

Salvage surgery was shown to be effective in recurrent cases after radiotherapy and partial resection. On the basis of these findings, we have established a treatment strategy for hypopharyngeal and laryngeal cancers. With prevention of neck and distant metastases representing a major issue, induction chemotherapy with CDDP plus 5-FU was given to cases of $\mathrm{T} 2$ or more advanced supraglottic cancer. Concurrent chemoradiotherapy was then performed on responders ( $\mathrm{PR}$ with improvement $\geq 80 \%$ and $\mathrm{CR}$ ), while surgery was selected for non-responders. For glottic cancer, since local control is important and neck metastasis is not frequently seen, local treatment was prioritized and concurrent chemoradiotherapy was administered to T3 or more advanced cases. For subglottic and hypopharyngeal cancers, treatment strategies were based on the same concept for supraglottic cancer. Irrespective of the status of primary site, induction chemotherapy was performed in N2 or more advanced cases with neck metastasis, and concurrent chemoradiotherapy was administered to responders while neck lymphadenectomy was performed on non-responders.

According to these strategies, concurrent chemoradiotherapy was performed in 29 cases, and the efficacy of CR was observed in 23 cases. However, local relapse was observed in 4 cases, with total resection performed in 3 cases finally. One patient was not suitable for salvage surgery and died. In PD, one patient underwent SCL-CHEP and laryngeal function was preserved. In total, laryngeal function was preserved in 19 cases.

Nedaplatin, cis-diammineglycolatoplatium (9), was developed to display reduced nephrotoxicity, which is often a dose-limiting factor of cisplatin $(10,11)$ and also to offer higher anti-tumour activity than carboplatin (12). In phase II clinical studies, high efficacy against head and neck cancer has been reported (13). The dose-limiting factor for nedaplatin is said to be haematological toxicity, as is the case with carboplatin $(10,11,14)$.

Critical adverse events at grade III/IV included 8 cases of neutropenia and 2 case of stomatitis. Anaphylactic symptoms appeared $30 \mathrm{~min}$ after nedaplatin administration in 3 cases (rash and mild seizure, $\mathrm{n}=2$ each). Severe symptoms such as bronchial asthma did not appear, and they were only temporary. However, this agent, as well as docetaxel and platinum compounds, has the potential to cause anaphylactic symptoms and should be used with caution.

The main weakness of this study was the limited number of enrolled patients. As the majority of patients displayed T1 and T2 glottic cancer and good performance status was limited, sample size was unavoidable. This treatment option must not be adopted without first obtaining further data. Significantly, toxicity of this treatment appears tolerable for the majority of patients. In conclusion, it is certain that concurrent chemoradiotherapy is efficacious for treatment of hypopharyngeal and laryngeal cancers in terms of organ function preservation and disease control. Patients with treatment failure could be controlled by salvage surgery and they can still have a chance of organ-function preservation. Distant disease should be prevented in patients with advanced nodal disease. We thus submit that this study provides a new concept for laryngeal function-preserving treatment that should be considered for locally advanced hypopharyngeal and laryngeal cancer.

\section{References}

1. Krengli M, Policarpo M, Manfredda I, et al: Voice quality after treatment for tla glottic carcinoma - radiotherapy versus laser cordectomy. Acta Oncol 43: 284-289, 2004.

2. Luscher MS, Pedersen U and Johansen LV: Treatment outcome after laser excision of early glottic squamous cell carcinoma - a literature survey. Acta Oncol 40: 796-800, 2001.

3. Forastiere AA, Goepfert H, Maor M, et al: Concurrent chemotherapy and radiotherapy for organ preservation in advanced laryngeal cancer. N Engl J Med 349: 2091-2098, 2003.

4. Ferlito A, Silver CE, Howard DJ, Laccourreye O, Rinaldo A and Owen R: The role of partial laryngeal resection in current management of laryngeal cancer: a collective review. Acta Otolaryngol 120: 456-465, 2000. 
5. Laccourreye H, Laccourreye O, Weinstein G, Menard M and Brasnu D: Supracricoid laryngectomy with cricohyoidoepiglottopexy: a partial laryngeal procedure for glottic carcinoma. Ann Otol Rhinol Laryngol 99: 421-426, 1990.

6. Motamed M, Laccourreye O and Bradley PJ: Salvage conservation laryngeal surgery after irradiation failure for early laryngeal cancer. Laryngoscope 116: 451-455, 2006

7. Alcock CJ, Fowler JF, Haybittle JL, Hopewell JW, Rezvani M and Wiernik G: Salvage surgery following irradiation with different fractionation regimes in the treatment of carcinoma of the laryngo pharynx: experience gained from a British institute of radiology study. J Laryngol Otol 106: 147-153, 1992.

8. Fowler BZ, Muller S, Chen AY and Johnstone PA: Factors influencing long-term survival following salvage total laryngectomy after initial radiotherapy or conservative surgery. Head Neck 28: 99-106, 2006.

9. Weiss RB and Christian MC: New cisplatin analogues in development. A review. Drugs 46: 360-377, 1993.

10. Kameyama Y, Okazaki N, Nakagawa M, Koshida H, Nakamura $\mathrm{M}$ and Gemba M: Nephrotoxicity of a new platinum compound, 254-s, evaluated with rat kidney cortical slices. Toxicol Lett 52: 15-24, 1990.
11. Suzumura Y, Kato T, Ueda R and Ota K: Effect of treatment schedule on antitumor activity of glycolate- 0,0 '-diammineplatinum(ii), a new platinum derivative: comparison with cisdiamminedichloroplatinum(ii). Anticancer Res 9: 1083-1088, 1989.

12. Kanzawa F, Matsushima Y, Nakano H, et al: Antitumor activity of a new platinum compound (glycolate-o,o') diammineplatinum (ii) (254-s), against non-small cell lung carcinoma grown in a human tumor clonogenic assay system. Anticancer Res 8: 323-327, 1988

13. Akaza H, Togashi M, Nishio Y, et al: Phase II study of cisdiammine(glycolato)platinum, 254-s, in patients with advanced germ-cell testicular cancer, prostatic cancer, and transitional-cell carcinoma of the urinary tract. 254-s urological cancer study group. Cancer Chemother Pharmacol 31: 187-192, 1992.

14. Hartmann JT and Lipp HP: Toxicity of platinum compounds. Expert Opin Pharmacother 4: 889-901, 2003. 